\title{
GEOMETRIC AND APPROXIMATION PROPERTIES OF SOME SINGULAR INTEGRALS IN THE UNIT DISK
}

\author{
GEORGE A. ANASTASSIOU AND SORIN G. GAL
}

Received 23 January 2006; Revised 19 April 2006; Accepted 20 April 2006

The purpose of this paper is to prove several results in approximation by complex Picard, Poisson-Cauchy, and Gauss-Weierstrass singular integrals with Jackson-type rate, having the quality of preservation of some properties in geometric function theory, like the preservation of coefficients' bounds, positive real part, bounded turn, starlikeness, and convexity. Also, some sufficient conditions for starlikeness and univalence of analytic functions are preserved.

Copyright (c) 2006 G. A. Anastassiou and S. G. Gal. This is an open access article distributed under the Creative Commons Attribution License, which permits unrestricted use, distribution, and reproduction in any medium, provided the original work is properly cited.

\section{Introduction}

Let us consider the open unit disk $D=\{z \in \mathbb{C} ;|z|<1\}$ and $A(\bar{D})=\{f: \bar{D} \rightarrow \mathbb{C} ; f$ is analytic on $D$, continuous on $\left.\bar{D}, f(0)=0, f^{\prime}(0)=1\right\}$. Therefore, if $f \in A(\bar{D})$, we have $f(z)=z+\sum_{k=2}^{\infty} a_{k} z^{k}$, for all $z \in D$.

For $f \in A(\bar{D})$ and $\xi \in \mathbb{R}, \xi>0$, let us consider the complex singular integrals

$$
\begin{gathered}
P_{\xi}(f)(z)=\frac{1}{2 \xi} \int_{-\infty}^{+\infty} f\left(z e^{i u}\right) e^{-|u| / \xi} d u, \quad z \in \bar{D} \\
Q_{\xi}(f)(z)=\frac{\xi}{\pi} \int_{-\pi}^{\pi} \frac{f\left(z e^{i u}\right)}{u^{2}+\xi^{2}} d u, \quad z \in \bar{D}, \quad Q_{\xi}^{*}(f)(z)=\frac{\xi}{\pi} \int_{-\infty}^{+\infty} \frac{f\left(z e^{-i u}\right)}{u^{2}+\xi^{2}} d u, \quad z \in \bar{D}, \\
R_{\xi}(f)(z)=\frac{2 \xi^{3}}{\pi} \int_{-\infty}^{+\infty} \frac{f\left(z e^{i u}\right)}{\left(u^{2}+\xi^{2}\right)^{2}} d u, \quad z \in \bar{D}, \\
W_{\xi}(f)(z)=\frac{1}{\sqrt{\pi \xi}} \int_{-\pi}^{\pi} f\left(z e^{i u}\right) e^{-u^{2} / \xi} d u, \quad z \in \bar{D}, \\
W_{\xi}^{*}(f)(z)=\frac{1}{\sqrt{\pi \xi}} \int_{-\infty}^{+\infty} f\left(z e^{-i u}\right) e^{-u^{2} / \xi} d u, \quad z \in \bar{D} .
\end{gathered}
$$


2 Geometric and approximation properties

Here $P_{\xi}(f)$ is said to be of Picard type, $Q_{\xi}(f), Q_{\xi}^{*}(f)$, and $R_{\xi}(f)$ are said to be of PoissonCauchy type, and $W_{\xi}(f)$ and $W_{\xi}^{*}(f)$ are said to be of Gauss-Weierstrass type.

In the very recent papers [3-5], classes of convolution complex polynomials were introduced and their approximation properties regarding rates, global smoothness preservation properties, and some geometric properties like the preservation of coefficients' bounds, positivity of real part, bounded turn, starlikeness, convexity, and univalence were proved.

The aim of this paper is to obtain similar properties for the above-defined complex singular integrals.

\section{Complex Picard integrals}

In this section, we study the properties of $P_{\xi}(f)(z)$.

Firstly, we present the approximation properties.

Theorem 2.1. Let $f \in A(\bar{D})$ and $\xi \in \mathbb{R}, \xi>0$. Then

(i) $P_{\xi}(f)(z)$ is continuous on $\bar{D}$, analytic on $D$, and $P_{\xi}(f)(0)=0$;

(ii) $\omega_{1}\left(P_{\xi}(f) ; \delta\right)_{\bar{D}} \leq \omega_{1}(f ; \delta)_{\bar{D}}$, for all $\delta \geq 0$, where $\omega_{1}(f ; \delta)_{\bar{D}}=\sup \left\{\left|f\left(z_{1}\right)-f\left(z_{2}\right)\right|\right.$; $\left.z_{1}, z_{2} \in \bar{D},\left|z_{1}-z_{2}\right| \leq \delta\right\}$;

(iii) $\left|P_{\xi}(f)(z)-f(z)\right| \leq C \omega_{2}(f ; \xi)_{\partial D}$, for all $z \in \bar{D}, \xi>0$, where

$$
\omega_{2}(f ; \xi)_{\partial D}=\sup \left\{\left|f\left(e^{i(x+u)}\right)-2 f\left(e^{i u}\right)+f\left(e^{i(x-u)}\right)\right| ; x \in \mathbb{R},|u| \leq \xi\right\} .
$$

Proof. (i) Let $z_{0}, z_{n} \in \bar{D}$ be with $\lim _{n \rightarrow \infty} z_{n}=z_{0}$. We get

$$
\begin{aligned}
\left|P_{\xi}(f)\left(z_{n}\right)-P_{\xi}(f)\left(z_{0}\right)\right| & \leq \frac{1}{2 \xi} \int_{-\infty}^{+\infty}\left|f\left(z_{n} e^{i u}\right)-f\left(z_{0} e^{i u}\right)\right| e^{-|u| / \xi} d u \\
& \leq \frac{1}{2 \xi} \int_{-\infty}^{+\infty} \omega_{1}\left(f ;\left|z_{n} e^{i u}-z_{0} e^{i u}\right|\right)_{\bar{D}} e^{-|u| / \xi} d u \\
& =\frac{1}{2 \xi} \int_{-\infty}^{+\infty} \omega_{1}\left(f ;\left|z_{n}-z_{0}\right|\right)_{\bar{D}} e^{-|u| / \xi} d u \\
& =\omega_{1}\left(f ;\left|z_{n}-z_{0}\right|\right)_{\bar{D}} .
\end{aligned}
$$

Passing to limit with $n \rightarrow \infty$, it follows that $P_{\xi}(f)(z)$ is continuous at $z_{0} \in \bar{D}$, since $f$ is continuous on $\bar{D}$. It remains to prove that $P_{\xi}(f)(z)$ is analytic on $D$. For $f \in A(\bar{D})$, we can write $f(z)=\sum_{k=0}^{\infty} a_{k} z^{k}, z \in D$. For fixed $z \in D$, we get $f\left(z e^{i u}\right)=\sum_{k=0}^{\infty} a_{k} e^{i k u} z^{k}$ and since $\left|a_{k} e^{i k u}\right|=\left|a_{k}\right|$, for all $u \in \mathbb{R}$, and the series $\sum_{k=0}^{\infty} a_{k} z^{k}$ is absolutely convergent, it follows that the series $\sum_{k=0}^{\infty} a_{k} e^{i k u} z^{k}$ is uniformly convergent with respect to $u \in \mathbb{R}$. This immediately implies that the series can be integrated term by term, that is,

$$
P_{\xi}(f)(z)=\frac{1}{2 \xi} \sum_{k=0}^{\infty} a_{k} z^{k}\left(\int_{-\infty}^{\infty} e^{i k u} e^{-|u| / \xi} d u\right)
$$

Also, since $a_{0}=0$, we get $P_{\xi}(f)(0)=0$. 
(ii) Let $z_{1}, z_{2} \in \bar{D},\left|z_{1}-z_{2}\right| \leq \delta$. We get

$$
\begin{aligned}
\left|P_{\xi}(f)\left(z_{1}\right)-P_{\xi}(f)\left(z_{2}\right)\right| & \leq \frac{1}{2 \xi} \int_{-\infty}^{+\infty}\left|f\left(z_{1} e^{i u}\right)-f\left(z_{2} e^{i u}\right)\right| e^{-|u| / \xi} d u \\
& \leq \omega_{1}\left(f ;\left|z_{1}-z_{2}\right|\right)_{\bar{D}} \leq \omega_{1}(f ; \delta)_{\bar{D}} .
\end{aligned}
$$

Passing to sup with $\left|z_{1}-z_{2}\right|<\delta$, the desired inequality follows.

(iii) We have

$$
\begin{aligned}
P_{\xi}(f)(z)-f(z) & =\frac{1}{2 \xi} \int_{-\infty}^{+\infty}\left[f\left(z e^{i u}\right)-f(z)\right] e^{-|u| / \xi} d u \\
& =\frac{1}{2 \xi} \int_{0}^{\infty}\left[f\left(z e^{i u}\right)-2 f(z)+f\left(z e^{-i u}\right)\right] e^{-u / \xi} d u,
\end{aligned}
$$

which implies

$$
\left|P_{\xi}(f)(z)-f(z)\right| \leq \frac{1}{2 \xi} \int_{0}^{\infty}\left|f\left(z e^{i u}\right)-2 f(z)+f\left(z e^{-i u}\right)\right| e^{-u / \xi} d u
$$

for all $z \in \bar{D}$.

By the maximum modulus principle (see, e.g., [3, page 421]), we can take $|z|=1$, case when

$$
\left|f\left(z e^{i u}\right)-2 f(z)+f\left(z e^{-i u}\right)\right| \leq \omega_{2}(f ; u)_{\partial D}
$$

which implies that for all $z \in \bar{D}$ we have

$$
\begin{aligned}
\left|P_{\xi}(f)(z)-f(z)\right| & \leq \frac{1}{2 \xi} \int_{0}^{+\infty} \omega_{2}(f ; u)_{\partial D} e^{-u / \xi} d u \\
& =\frac{1}{2 \xi} \int_{0}^{+\infty} \omega_{2}\left(f ; \frac{u}{\xi} \cdot \xi\right)_{\partial D} e^{-u / \xi} d u \\
& \leq\left(\frac{1}{2 \xi} \int_{0}^{+\infty}\left[1+\frac{u}{\xi}\right]^{2} e^{-u / \xi} d u\right) \omega_{2}(f ; \xi)_{\partial D} \leq C \omega_{2}(f ; \xi)_{\partial D}
\end{aligned}
$$

(for the last inequalities, see, e.g., [2, proof of Theorem 2.1(i), page 252]).

Remark 2.2. Theorem 2.1(ii) and (iii) remain valid for $f$ only continuous on $\bar{D}$.

In what follows, we present some geometric properties of $P_{\xi}(f)(z)$.

Theorem 2.3. If $f(z)=\sum_{k=0}^{\infty} a_{k} z^{k}$, for all $z \in D$, then

$$
P_{\xi}(f)(z)=\sum_{k=0}^{\infty} \frac{a_{k}}{1+\xi^{2} k^{2}} z^{k}
$$


4 Geometric and approximation properties

for all $z \in D$, that is, if $f(0)=0$, then $P_{\xi}(f)(0)=0$ and if $f^{\prime}(0)=1$, then $P_{\xi}^{\prime}(f)(0)=$ $1 /\left(1+\xi^{2}\right) \neq 1$, for all $\xi>0$. Also,

$$
\left|a_{k}\left(P_{\xi}(f)\right)\right|=\left|\frac{a_{k}(f)}{1+\xi^{2} k^{2}}\right| \leq\left|a_{k}(f)\right|, \quad \forall k=0,1, \ldots
$$

Proof. In the proof of Theorem 2.1(i), we can write

$$
P_{\xi}(f)(z)=\sum_{k=0}^{\infty} a_{k} z^{k}\left[\frac{1}{2 \xi} \int_{-\infty}^{+\infty} e^{i k u} e^{-|u| / \xi} d u\right], \quad \forall z \in D .
$$

But

$$
\begin{aligned}
& \frac{1}{2 \xi} \int_{-\infty}^{+\infty} e^{i k u} e^{-|u| / \xi} d u \\
& \quad=\frac{1}{2 \xi} \int_{-\infty}^{+\infty} \cos (k u) \cdot e^{-|u| / \xi} d u=\frac{1}{\xi} \int_{0}^{+\infty} \cos (k u) e^{-u / \xi} d u \\
& \quad=\left.\frac{1}{\xi} \cdot \frac{e^{-u / \xi}[-(1 / \xi) \cos (k u)+k \sin (k u)]}{1 / \xi^{2}+k^{2}}\right|_{0} ^{\infty}=\frac{1}{1+k^{2} \xi^{2}},
\end{aligned}
$$

which proves the theorem.

Now, recall that a function $f \in A(\bar{D})$ is starlike if it is univalent and $f(D)$ is a starlike plane domain with respect to 0 , and is convex if it is univalent on $D$ and $f(D)$ is a convex plane domain.

Also, let us introduce the following classes of analytic functions:

$$
\begin{gathered}
S_{1}=\left\{f \in A(\bar{D}) ; f(z)=z+\sum_{k=2}^{\infty} a_{k} z^{k}, \sum_{k=2}^{\infty} k\left|a_{k}\right| \leq 1\right\}, \\
S_{2}=\left\{f \text { analytic in } D, f(z)=\sum_{k=1}^{\infty} a_{k} z^{k}, z \in D,\left|a_{1}\right| \geq \sum_{k=2}^{\infty}\left|a_{k}\right|\right\}, \\
S_{3}=\left\{f \in A(\bar{D}) ;\left|f^{\prime \prime}(z)\right| \leq 1, \forall z \in D\right\}, \\
\mathscr{P}=\{f: \bar{D} \longrightarrow \mathbb{C} ; f \text { is analytic on } D, f(0)=1, \operatorname{Re}[f(z)]>0, \forall z \in D\}, \\
\mathscr{R}=\left\{f \in A(\bar{D}) ; \operatorname{Re}\left[f^{\prime}(z)\right]>0, \forall z \in D\right\}, \\
S_{M}=\left\{f \in A(\bar{D}) ;\left|f^{\prime}(z)\right|<M, \forall z \in D\right\}, \quad M>1 .
\end{gathered}
$$

According to, for example, [6, Exercise 4.9.1, page 97], if $f \in S_{1}$, then $\left|z f^{\prime \prime}(z) / f(z)-1\right|<$ 1 , for all $z \in D$, and therefore $f$ is starlike (and univalent) on $D$.

According to [1, page 22 ], if $f \in S_{2}$, then $f$ is starlike (and univalent) on $D$.

By [7], if $f \in S_{3}$, then $f$ is starlike (and univalent) on $D$. Also, it is well known that $\mathscr{R}$ is the class of functions with bounded turn (i.e., $\left|\arg f^{\prime}(z)\right|<\pi / 2$, for all $z \in D$ ) and that $f \in \mathscr{R}$ implies the univalency of $f$ on $D$.

According to, for example, [6, Exercise 5.4.1, page 111], $f \in S_{M}$ implies that $f$ is univalent in $\{z \in \mathbb{C} ;|z|<1 / M\}$. 
We present the following.

Theorem 2.4. For all $\xi>0$,

$$
P_{\xi}\left(S_{2}\right) \subset S_{2}, \quad P_{\xi}(\mathscr{P}) \subset \mathscr{P}
$$

Proof. By Theorem 2.3, for $f(z)=\sum_{k=1}^{\infty} a_{k} z^{k} \in S_{2}$, we get

$$
\sum_{k=2}^{\infty}\left|\frac{a_{k}}{1+\xi^{2} k^{2}}\right|=\sum_{k=2}^{\infty} \frac{\left|a_{k}\right|}{1+\xi^{2}} \cdot \frac{1+\xi^{2}}{1+\xi^{2} k^{2}} \leq \frac{1}{1+\xi^{2}} \sum_{k=2}^{\infty}\left|a_{k}\right| \leq \frac{\left|a_{1}\right|}{1+\xi^{2}}
$$

and since $P_{\xi}(f)(z)=\sum_{k=0}^{\infty}\left(a_{k} /\left(1+\xi^{2} k^{2}\right)\right) z^{k}$, it follows that $P_{\xi}(f) \in S_{2}$.

Let $f(z)=\sum_{k=0}^{\infty} a_{k} z^{k} \in \mathscr{P}$, that is, $a_{0}=1$ and if $f(z)=U(x, y)+i V(x, y), z=x+i y \in D$, then $U(x, y)>0$, for all $z=x+i y \in D$.

We get $P_{\xi}(f)(0)=a_{0}=1$ and

$$
\begin{aligned}
P_{\xi}(f)(z)= & \frac{1}{2 \xi} \int_{-\infty}^{+\infty} U(r \cos (u+t), r \sin (u+t)) e^{-|u| / \xi} d u \\
& +i \cdot \frac{1}{2 \xi} \int_{-\infty}^{+\infty} V(r \cos (u+t), r \sin (u+t)) e^{-|u| / \xi} d u, \quad \forall z=r e^{i t} \in D,
\end{aligned}
$$

which immediately implies

$$
\operatorname{Re}\left[P_{\xi}(f)(z)\right]=\frac{1}{2 \xi} \int_{-\infty}^{+\infty} U(r \cos (u+t), r \sin (u+t)) e^{-|u| / \xi} d u>0
$$

that is, $P_{\xi}(f) \in \mathscr{P}$.

Theorem 2.5. For all $\xi>0,\left(1+\xi^{2}\right) P_{\xi}\left(S_{1}\right) \subset S_{1},\left(1+\xi^{2}\right) P_{\xi}\left(S_{M}\right) \subset S_{M\left(1+\xi^{2}\right)}$, and $(1+$ $\left.\xi^{2}\right) P_{\xi}\left(S_{3, \xi}\right) \subset S_{3}$, where

$$
S_{3, \xi}=\left\{f \in S_{3} ;\left|f^{\prime \prime}(z)\right| \leq \frac{1}{1+\xi^{2}}, \forall z \in D\right\} \subset S_{3} .
$$

Proof. Let $f \in S_{1}$. By Theorem 2.3, we obtain

$$
\left(1+\xi^{2}\right) P_{\xi}(f)(z)=\sum_{k=1}^{\infty} a_{k} \frac{1+\xi^{2}}{1+\xi^{2} k^{2}} z^{k}
$$

if $f(z)=\sum_{k=1}^{\infty} a_{k} z^{k} \in S_{1}$. It follows that $\left(1+\xi^{2}\right) P_{\xi}^{\prime}(f)(0)=a_{1}=1$, that is,

$$
\begin{gathered}
\left(1+\xi^{2}\right) P_{\xi}(f)(z)=z+\sum_{k=2}^{\infty} a_{k} \cdot \frac{1+\xi^{2}}{1+\xi^{2} k^{2}} z^{k}, \\
\sum_{k=2}^{\infty} k\left|a_{k}\right| \frac{1+\xi^{2}}{1+\xi^{2} k^{2}} \leq \sum_{k=2}^{\infty} k\left|a_{k}\right| \leq 1,
\end{gathered}
$$

that is, $\left(1+\xi^{2}\right) P_{\xi}(f) \in S_{1}$. 
Let $f \in S_{M}$. We get

$$
\begin{aligned}
\left|\left(1+\xi^{2}\right) P_{\xi}^{\prime}(f)(z)\right| & =\left(1+\xi^{2}\right) \cdot\left|\frac{1}{2 \xi} \int_{-\infty}^{+\infty} f^{\prime}\left(z e^{i u}\right) e^{i u} e^{-|u| / \xi} d u\right| \\
& \leq\left(1+\xi^{2}\right) \frac{1}{2 \xi} \int_{-\infty}^{+\infty}\left|f^{\prime}\left(z e^{i u}\right)\right| e^{-|u| / \xi} d u<M\left(1+\xi^{2}\right), \quad z \in D .
\end{aligned}
$$

Also, $P_{\xi}(f)(0)=0$ and $\left(1+\xi^{2}\right) P_{\xi}^{\prime}(f)(0)=1$, which implies that $\left(1+\xi^{2}\right) P_{\xi}(f) \in S_{M\left(1+\xi^{2}\right)}$.

Now, let $f \in S_{3, \xi}$. We have

$$
\left(1+\xi^{2}\right) P_{\xi}^{\prime \prime}(f)(z)=\left(1+\xi^{2}\right) \cdot \frac{1}{2 \xi} \int_{-\infty}^{+\infty} f^{\prime \prime}\left(z e^{i u}\right) e^{2 i u} e^{-|u| / \xi} d u
$$

which implies

$$
\left|\left(1+\xi^{2}\right) P_{\xi}^{\prime \prime}(f)(z)\right| \leq\left(1+\xi^{2}\right) \frac{1}{2 \xi} \cdot \int_{-\infty}^{+\infty}\left|f^{\prime \prime}\left(z e^{i u}\right)\right| e^{-|u| / \xi} d u \leq 1,
$$

that is, $\left(1+\xi^{2}\right) P_{\xi}(f) \in S_{3}$.

Remarks 2.6. (1) Since the constant $\left(1+\xi^{2}\right)$ does not influence the geometric properties of $P_{\xi}(f)$, it follows that for all $\xi>0$ we have the following:

(i) if $f \in S_{1}$, then $P_{\xi}(f)$ is starlike (and univalent) in $D$;

(ii) if $f \in S_{M}$, then $P_{\xi}(f)$ is univalent in $\left\{z \in \mathbb{C}\right.$; $\left.|z|<1 / M\left(1+\xi^{2}\right)\right\}$;

(iii) if $f \in S_{3, \xi} \subset S_{3}$, then $P_{\xi}(f)$ is starlike and univalent in $D$.

(2) Since

$$
P_{\xi}^{\prime}(f)(z)=\frac{1}{2 \xi} \int_{-\infty}^{+\infty} f^{\prime}\left(z e^{i u}\right) e^{i u} e^{-|u| / \xi} d u
$$

it is obvious that the condition $\operatorname{Re}\left[f^{\prime}(z)\right]>0$, for all $z \in D$, does not imply $\operatorname{Re}\left[P_{\xi}^{\prime}(f)(z)\right]>$ 0 on $D$.

In this case, we may follow the idea in, for example, [5, Theorem 3.4] to construct another singular integral as follows: for $f \in A(\bar{D})$, we define $S_{\xi}(f)(z)=\int_{0}^{z} Q_{n}(u) d u$ with

$$
Q_{n}(z)=\frac{1}{2 \xi} \int_{-\infty}^{+\infty} f^{\prime}\left(z e^{i t}\right) e^{-|t| / \xi} d t
$$

Then, it is an easy task to show that $\left(1+\xi^{2}\right) S_{\xi}(\mathscr{R}) \subset \mathscr{R}$, for all $\xi>0$, and the following estimate holds:

$$
\left|S_{\xi}(f)(z)-f(z)\right| \leq C \omega_{2}\left(f^{\prime} ; \xi\right)_{\partial D}, \quad \forall z \in D, \xi>0 .
$$

Since $\inf \left\{1 /\left(1+\xi^{2}\right) ; \xi \in[0,1]\right\}=1 / 2$, by Theorem 2.5 , the following is immediate.

Corollary 2.7. $P_{\xi}\left(S_{3,1 / 2}\right) \subset S_{3}$ and $f \in S_{M}$ implies that $P_{\xi}(f)$ is univalent in $\{z \in \mathbb{C} ;|z|<$ $1 / 2 M\}$, for all $\xi \in[0,1]$. 
Remark 2.8. Of course, if we consider, for example, $\xi \in[0,1 / 2]$, then $\inf \left\{1 /\left(1+\xi^{2}\right) ; x \in\right.$ $[0,1 / 2]\}=4 / 5$ and by Theorem 2.5 we get $P_{\xi}\left(S_{3}, 4 / 5\right) \subset S_{3}$ and $f \in S_{M}$ implies that $P_{\xi}(f)$ is univalent in $\{z \in \mathbb{C} ;|z|<4 / 5 M\}$, for all $\xi \in[0,1 / 2]$.

Obviously $S_{3,1 / 2} \subset S_{3,5 / 4}$ and $\{z \in \mathbb{C} ;|z|<1 / 2 M\} \subset\{z \in \mathbb{C} ;|z|<4 / 5 M\}$.

\section{Complex Poisson-Cauchy integrals}

In this section, we study the properties of $Q_{\xi}(f), Q_{\xi}^{*}(f)$, and $R_{\xi}(f)$.

Firstly, we present the approximation properties.

Theorem 3.1. (i) If $f(z)=\sum_{k=0}^{\infty} a_{k} z^{k}$ is analytic in $D$, then for all $\xi>0, Q_{\xi}(f)(z)$, $Q_{\xi}^{*}(f)(z)$, and $R_{\xi}(f)(z)$ are analytic in $D$ and the following hold in $D$ :

$$
\begin{gathered}
Q_{\xi}(f)(z)=\sum_{k=0}^{\infty} a_{k} b_{k}(\xi) z^{k}, \quad \text { with } b_{k}(\xi)=\frac{2 \xi}{\pi} \int_{0}^{\pi} \frac{\cos k u}{u^{2}+\xi^{2}} d u, \\
Q_{\xi}^{*}(f)(z)=\sum_{k=0}^{\infty} a_{k} b_{k}^{*}(\xi) z^{k}, \quad \text { with } b_{k}^{*}(\xi)=\frac{2 \xi}{\pi} \int_{0}^{+\infty} \frac{\cos k u}{u^{2}+\xi^{2}} d u, \\
R_{\xi}(f)(z)=\sum_{k=0}^{\infty} a_{k} c_{k}(\xi) z^{k}, \quad \text { with } c_{k}(\xi)=\frac{4 \xi^{3}}{\pi} \int_{0}^{\infty} \frac{\cos k u}{\left(u^{2}+\xi^{2}\right)^{2}} d u .
\end{gathered}
$$

Also, if $f$ is continuous on $\bar{D}$, then $Q_{\xi}(f), Q_{\xi}^{*}(f)$, and $R_{\xi}(f)$ are also continuous on $\bar{D}$.

Here $b_{1}(\xi)>0$, for all $\xi>0, b_{1}^{*}(\xi)=e^{-\xi}, c_{1}(\xi)=(1+\xi) e^{-\xi}$, for all $\xi>0$.

(ii)

$$
\begin{array}{ll}
\left|Q_{\xi}(f)(z)-f(z)\right| \leq C \frac{\omega_{2}(f ; \xi)_{\partial D}}{\xi}, & \forall x \in \bar{D}, \xi \in(0,1], \\
\left|Q_{\xi}^{*}(f)(z)-f(z)\right| \leq C \frac{\omega_{2}(f ; \xi)_{\partial D}}{\xi}, \quad \forall z \in \bar{D}, \xi \in(0,1], \\
\left|R_{\xi}(f)(z)-f(z)\right| \leq C \omega_{1}(f ; \xi)_{\bar{D}}, \quad \forall z \in \bar{D}, \xi \in(0,1] .
\end{array}
$$

(iii)

$$
\begin{array}{cc}
\omega_{1}\left(Q_{\xi}^{*}(f) ; \delta\right)_{\bar{D}} \leq \omega_{1}(f ; \delta)_{\bar{D}}, & \forall \xi \in(0,1], \delta>0, \\
\omega_{1}\left(Q_{\xi}(f) ; \delta\right)_{\bar{D}} \leq \omega_{1}(f ; \delta)_{\bar{D}}, & \forall \xi \in(0,1], \forall \delta>0, \\
\omega_{1}\left(R_{\xi}(f) ; \delta\right)_{\bar{D}} \leq \omega_{1}(f ; \delta)_{\bar{D}}, & \forall \xi \in(0,1], \delta>0 .
\end{array}
$$

Proof. (i) Let $f(z)=\sum_{k=0}^{\infty} a_{k} z^{k}, z \in D$.

Reasoning as for the case of Picard-type integral in Theorem 2.1(i), we obtain

$$
Q_{\xi}(f)(z)=\sum_{k=0}^{\infty} a_{k} z^{k}\left[\frac{\xi}{\pi} \int_{-\pi}^{\pi} e^{i k u} \cdot \frac{1}{u^{2}+\xi^{2}} d u\right]
$$


8 Geometric and approximation properties

where

$$
\begin{aligned}
\frac{\xi}{\pi} \int_{-\pi}^{\pi} e^{i k u} \cdot \frac{1}{u^{2}+\xi^{2}} d u & =\frac{\xi}{\pi} \int_{-\pi}^{\pi} \frac{\cos k u}{u^{2}+\xi^{2}} d u+i \frac{\xi}{\pi} \int_{-\pi}^{\pi} \frac{\sin k u}{u^{2}+\xi^{2}} d u \\
& =\frac{2 \xi}{\pi} \int_{0}^{\pi} \frac{\cos k u}{u^{2}+\xi^{2}} d u=b_{k}(\xi), \\
Q_{\xi}^{*}(f)(z) & =\sum_{k=0}^{\infty} a_{k} z^{k}\left[\frac{\xi}{\pi} \int_{-\infty}^{+\infty} e^{i k u} \cdot \frac{1}{u^{2}+\xi^{2}} d u\right],
\end{aligned}
$$

where

$$
\begin{gathered}
\frac{\xi}{\pi} \int_{-\infty}^{+\infty} e^{i k u} \cdot \frac{1}{u^{2}+\xi^{2}} d u=\frac{2 \xi}{\pi} \int_{0}^{\infty} \frac{\cos k u}{u^{2}+\xi^{2}} d u=b_{k}^{*}(\xi), \\
R_{\xi}(f)(z)=\sum_{k=0}^{\infty} a_{k} z^{k}\left[\frac{2 \xi^{3}}{\pi} \int_{-\infty}^{+\infty} \frac{e^{i k u}}{\left(u^{2}+\xi^{2}\right)^{2}} d u\right]
\end{gathered}
$$

where

$$
\frac{2 \xi^{3}}{\pi} \int_{-\infty}^{+\infty} e^{i k u} \cdot \frac{1}{\left(u^{2}+\xi^{2}\right)^{2}} d u=\frac{4 \xi^{3}}{\pi} \int_{0}^{\infty} \frac{\cos k u}{\left(u^{2}+\xi^{2}\right)^{2}} d u
$$

The continuity of $f$ on $\bar{D}$ implies the continuity of $Q_{\xi}(f), Q_{\xi}^{*}(f)$, and $R_{\xi}(f)$ as in the proof of Theorem 2.1(i) for $P_{\xi}(f)$.

It remains to show that $b_{1}(\xi)>0$ and $b_{1}^{*}(\xi)=e^{-\xi}, c_{1}(\xi)=(1+\xi) e^{-\xi}$, for all $\xi>0$. Indeed, firstly we have

$$
\begin{aligned}
b_{1}(\xi) & =\frac{2 \xi}{\pi} \int_{0}^{\pi} \frac{\cos u}{u^{2}+\xi^{2}} d u=\frac{2 \xi}{\pi}\left[\int_{0}^{\pi / 2} \frac{\cos u}{u^{2}+\xi^{2}} d u+\int_{\pi / 2}^{\pi} \frac{\cos u}{u^{2}+\xi^{2}} d u\right] \\
& =\frac{2 \xi}{\pi}\left[\int_{0}^{\pi / 2} \frac{\cos u}{u^{2}+\xi^{2}} d u-\int_{0}^{\pi / 2} \frac{\sin u}{(u+\pi / 2)^{2}+\xi^{2}} d u\right] \\
& >\frac{2 \xi}{\pi} \int_{0}^{\pi / 2} \frac{\cos u-\sin u}{u^{2}+\xi^{2}} d u \\
& =\frac{2 \xi}{\pi}\left[\int_{0}^{\pi / 4} \frac{\cos u-\sin u}{u^{2}+\xi^{2}} d u+\int_{\pi / 4}^{\pi / 2} \frac{\cos u-\sin u}{u^{2}+\xi^{2}} d u\right]:=\frac{2 \xi}{\pi}\left[I_{1}+I_{2}\right] .
\end{aligned}
$$

Here

$$
\begin{aligned}
0 & <I_{1}=\int_{0}^{\pi / 4} \frac{\cos u-\sin u}{u^{2}+\xi^{2}} d u>\int_{0}^{\pi / 4} \frac{\cos u-\sin u}{\left(\pi^{2} / 16\right)+\xi^{2}} d u \\
& =\frac{16}{\pi^{2}+16 \xi^{2}}[\sin u+\cos u]_{0}^{\pi / 4}=\frac{16(\sqrt{2}-1)}{\pi^{2}+16 \xi^{2}} .
\end{aligned}
$$


Also, $I_{2}<0$ and

$$
\begin{aligned}
\left|I_{2}\right| & =-I_{2}=\int_{\pi / 4}^{\pi / 2} \frac{\sin u-\cos u}{u^{2}+\xi^{2}} d u \leq \frac{1}{\left(\pi^{2} / 16\right)+\xi^{2}} \cdot \int_{\pi / 4}^{\pi / 2}[\sin u-\cos u] d u \\
& =\frac{16}{\pi^{2}+16 \xi^{2}}[-\cos u-\sin u]_{\pi / 4}^{\pi / 2}=\frac{16(\sqrt{2}-1)}{\pi^{2}+16 \xi^{2}},
\end{aligned}
$$

which implies $I_{1}+I_{2} \geq 0$. Therefore, it follows that $b_{1}(\xi)>(2 \xi / \pi)\left[I_{1}+I_{2}\right] \geq 0$, for all $\xi>0$. Now let

$$
b_{1}^{*}(\xi)=\frac{2 \xi}{\pi} \int_{0}^{\infty} \frac{\cos u}{u^{2}+\xi^{2}} d u=\left(\text { by } v=\frac{u}{\xi}\right)=\frac{2}{\pi} \cdot \int_{0}^{\infty} \frac{\cos (u \xi)}{u^{2}+1} d u .
$$

Applying now the classical residue theorem to $f(z)=e^{i z} /\left(z^{2}+1\right)$, it is immediate that $\int_{0}^{\infty}\left(\cos (u \xi) /\left(u^{2}+1\right)\right) d u=(\pi / 2) e^{-\xi}$, which implies $b_{1}^{*}(\xi)=(2 / \pi) \cdot(\pi / 2) e^{-\xi}=e^{-\xi}$, for all $\xi>0$. For $c_{1}(\xi)=\left(4 \xi^{3} / \pi\right) \cdot \int_{0}^{\infty}\left(\cos u /\left(u^{2}+\xi^{2}\right)^{2}\right) d u$, applying the residue theorem to $f(z)=$ $e^{i z} /\left(z^{2}+\xi^{2}\right)^{2}$, we immediately get

$$
\int_{0}^{\infty} \frac{\cos u}{\left(u^{2}+\xi^{2}\right)^{2}} d u=\frac{\pi}{4 \xi^{3}}(1+\xi) e^{-\xi}
$$

that is, $c_{1}(\xi)=(1+\xi) e^{-\xi}$, for all $\xi>0$.

(ii) We can write

$$
Q \xi(f)(z)-f(z)=\frac{\xi}{\pi} \int_{0}^{\pi} \frac{f\left(z e^{i u}\right)-2 f(z)+f\left(z e^{-i u}\right)}{u^{2}+\xi^{2}} d u-f(z) E(\xi),
$$

where

$$
|E(\xi)|=E(\xi)=1-\frac{2 \xi}{\pi} \int_{0}^{\pi} \frac{d u}{u^{2}+\xi^{2}}=1-\frac{2}{\pi} \operatorname{arctg} \frac{\pi}{\xi} \leq \frac{2}{\pi^{2}} \xi
$$

(for the last estimate $|E(\xi)| \leq\left(2 / \pi^{2}\right) \xi$, see, e.g., [2, page 257]).

Passing to modulus, it follows that

$$
\begin{aligned}
\left|Q_{\xi}(f)(z)-f(z)\right| & \leq \frac{\xi}{\pi} \int_{0}^{\pi} \frac{\left|f\left(z e^{i u}\right)-2 f(z)+f\left(z e^{-i u}\right)\right|}{u^{2}+\xi^{2}} d u+\|f\|_{\bar{D}}|E(\xi)| \\
& \leq \frac{\xi}{\pi} \int_{0}^{\pi} \frac{\omega_{2}(f ; u)_{\partial D}}{u^{2}+\xi^{2}} d u+\|f\|_{\bar{D}} \cdot|E(\xi)| \\
& \leq C \frac{\xi}{\pi} \cdot \omega_{2}(f ; \xi)_{\partial D} \cdot \int_{0}^{\pi}\left[1+\frac{u}{\xi}\right]^{2} \frac{1}{u^{2}+\xi^{2}} d u .
\end{aligned}
$$

Reasoning as in the proof of Theorem 3.1 [2, pages 257-258], we arrive at the desired estimate.

For $Q_{\xi}^{*}(f)(z)$, we have

$$
Q_{\xi}^{*}(f)(z)-f(z)=\frac{\xi}{\pi} \int_{0}^{\infty} \frac{\left[f\left(z e^{i u}\right)-2 f(z)+f\left(z e^{-i u}\right)\right]}{u^{2}+\xi^{2}} d u,
$$


which implies

$$
\begin{aligned}
\left|Q_{\xi}^{*}(f)(z)-f(z)\right| & \leq \frac{\xi}{\pi} \int_{0}^{\infty} \frac{\left|f\left(z e^{i u}\right)-2 f(z)+f\left(z e^{-i u}\right)\right|}{u^{2}+\xi^{2}} d u \\
& \leq C \frac{\xi}{\pi} \int_{0}^{\infty} \frac{\omega_{2}(f ; u)_{\partial D}}{u^{2}+\xi^{2}} d u=C \frac{\xi}{\pi} \int_{0}^{\infty} \frac{\omega_{2}(f ;(u / \xi) \cdot \xi)_{\partial D}}{u^{2}+\xi^{2}} d u \\
& \leq C \omega_{2}(f ; \xi)_{\partial D} \cdot \frac{\xi}{\pi} \int_{0}^{\infty}\left[1+\frac{u}{\xi}\right]^{2} \cdot \frac{1}{u^{2}+\xi^{2}} d u \leq C \frac{\omega_{2}(f ; \xi)_{\partial D}}{\xi}
\end{aligned}
$$

For $R_{\xi}(f)(z)$, we obtain

$$
\begin{aligned}
\left|R_{\xi}(f)(z)-f(z)\right| & \leq \frac{2 \xi^{3}}{\pi} \int_{-\infty}^{+\infty} \frac{\left|f\left(z e^{i u}\right)-f(z)\right|}{\left(u^{2}+\xi^{2}\right)^{2}} d u \\
& \leq \frac{2 \xi^{3}}{\pi} \int_{-\infty}^{+\infty} \frac{\omega_{1}\left(f ;|z| \cdot\left|e^{i u}-1\right|\right)_{\bar{D}}}{\left(u^{2}+\xi^{2}\right)^{2}} d u \\
& \leq C \frac{2 \xi^{3}}{\pi} \int_{-\infty}^{+\infty} \frac{\omega_{1}(f ;|u|)_{\bar{D}}}{\left(u^{2}+\xi^{2}\right)^{2}} d u \\
& \leq C \frac{2 \xi^{3}}{\pi} \int_{0}^{\infty} \omega_{1}\left(f ; \frac{u}{\xi} \cdot \xi\right)_{\bar{D}} \cdot \frac{1}{\left(u^{2}+\xi^{2}\right)^{2}} d u \\
& \leq C \omega_{1}(f ; \xi)_{\bar{D}} \frac{2 \xi^{3}}{\pi} \int_{0}^{\infty}\left[1+\frac{u}{\xi}\right] \cdot \frac{1}{\left(u^{2}+\xi^{2}\right)^{2}} d u \\
& =C \omega_{1}(f ; \xi)_{\bar{D}}\left[1+\frac{2 \xi^{2}}{\pi} \int_{0}^{\infty} \frac{u}{\left(u^{2}+\xi^{2}\right)^{2}} d u\right],
\end{aligned}
$$

where

$$
\frac{2 \xi^{2}}{\pi} \int_{0}^{\infty} \frac{u d u}{\left(u^{2}+\xi^{2}\right)^{2}}=\frac{2 \xi^{2}}{\pi} \cdot \frac{1}{2} \int_{\xi^{2}}^{\infty} \frac{d v}{v^{2}}=\left.\frac{\xi^{2}}{\pi} \cdot\left(-\frac{1}{v}\right)\right|_{\xi^{2}} ^{\infty}=\frac{1}{\pi}
$$

which proves the estimate for $R_{\xi}(f)(z)$ too.

(iii) Let $z_{1}, z_{2} \in \bar{D}$ be with $\left|z_{1}-z_{2}\right| \leq \delta$. We get

$$
\begin{aligned}
\left|Q_{\xi}^{*}(f)\left(z_{1}\right)-Q_{\xi}^{*}(f)\left(z_{2}\right)\right| & \leq \frac{\xi}{\pi} \int_{-\infty}^{+\infty} \frac{\left|f\left(z_{1} e^{i u}\right)-f\left(z_{2} e^{i u}\right)\right|}{u^{2}+\xi^{2}} d u \\
& \leq \omega_{1}\left(f ;\left|z_{1}-z_{2}\right|\right)_{\bar{D}} \frac{\xi}{\pi} \int_{-\infty}^{+\infty} \frac{d u}{u^{2}+\xi^{2}} \leq \omega_{1}(f ; \delta)_{\bar{D}}
\end{aligned}
$$

where from passing to supremum after $z_{1}, z_{2}$ it follows that $\omega_{1}\left(Q_{\xi}^{*}(f) ; \delta\right)_{\bar{D}} \leq \omega_{1}(f ; \delta)_{\bar{D}}$. 
Also

$$
\begin{aligned}
\left|Q_{\xi}(f)\left(z_{1}\right)-Q_{\xi}(f)\left(z_{2}\right)\right| & \leq \frac{\xi}{\pi} \int_{-\pi}^{\pi} \frac{\left|f\left(z_{1} e^{i u}\right)-f\left(z_{2} e^{i u}\right)\right|}{u^{2}+\xi^{2}} d u \\
& \leq \omega_{1}\left(f ;\left|z_{1}-z_{2}\right|\right)_{\bar{D}} \cdot \frac{\xi}{\pi} \int_{-\pi}^{\pi} \frac{d u}{u^{2}+\xi^{2}} \\
& \leq \omega_{1}(f ; \delta)_{\bar{D}} \cdot \frac{\xi}{\pi} \int_{-\infty}^{+\infty} \frac{d u}{u^{2}+\xi^{2}}=\omega_{1}(f ; \delta)_{\bar{D}}
\end{aligned}
$$

The reasonings for $R_{\xi}(f)$ are similar, which proves the theorem.

In what follows, we present some geometric properties of complex Poisson-Cauchy integrals.

Theorem 3.2. (i) If $f(z)=\sum_{k=0}^{\infty} a_{k} z^{k}, z \in D$, and $T_{\xi}(f)(z)=\sum_{k=0}^{\infty} A_{k} z^{k}$ is any from $Q_{\xi}(f)$, $Q_{\xi}^{*}(f)$, and $R_{\xi}(f)$, then

$$
\left|A_{k}\right| \leq\left|a_{k}\right|, \quad \forall k=0,1, \ldots
$$

(ii) If $f(z)=\sum_{k=1}^{\infty} a_{k} z^{k}, z \in D$, is univalent in $D$ and $f(D)$ is convex, then for any $\xi>0$, $Q_{\xi}(f)(z)$ is close-to-convex on D.

(iii) For all $\xi>0$, with the notation in Section $2, Q_{\xi}^{*}(\mathscr{P}) \subset \mathscr{P}, R_{\xi}(\mathscr{P}) \subset \mathscr{P}$;

$$
\begin{array}{cc}
\frac{1}{b_{1}(\xi)} \cdot Q_{\xi}\left(S_{3, b_{1}(\xi)}\right) \subset S_{3}, & \frac{1}{b_{1}^{*}(\xi)} \cdot Q_{\xi}^{*}\left(S_{3, b_{1}^{*}(\xi)}\right) \subset S_{3}, \\
\frac{1}{c_{1}(\xi)} \cdot R_{\xi}\left(S_{3, c_{1}(\xi)}\right) \subset S_{3}, & \frac{1}{b_{1}(\xi)} Q_{\xi}\left(S_{M}\right) \subset S_{M /\left|b_{1}(\xi)\right|}, \\
\frac{1}{b_{1}^{*}(\xi)} Q_{\xi}^{*}\left(S_{M}\right) \subset S_{M /\left|b_{1}^{*}(\xi)\right|}, & \frac{1}{c_{1}(\xi)} R_{\xi}\left(S_{M}\right) \subset S_{M /\left|c_{1}(\xi)\right|},
\end{array}
$$

where $S_{3, a}=\left\{f \in S_{3} ;\left|f^{\prime \prime}(z)\right| \leq|a|\right\}$ and $S_{B}=\left\{f \in A(\bar{D}) ;\left|f^{\prime}(z)\right|<B, z \in D\right\}$.

Proof. (i) With the notations in the statement of Theorem 3.1(i), for all $k=0,1,2, \ldots$, we obtain

$$
\begin{aligned}
\left|b_{k}(\xi)\right| & \leq \frac{2 \xi}{\pi} \int_{0}^{\pi} \frac{|\cos k u|}{u^{2}+\xi^{2}} d u \leq \frac{2 \xi}{\pi} \int_{0}^{\pi} \frac{d u}{u^{2}+\xi^{2}} \\
& =\left.\frac{2 \xi}{\pi} \cdot \frac{1}{\xi} \operatorname{arctg} \frac{u}{\xi}\right|_{0} ^{\pi}=\frac{2}{\pi} \operatorname{arctg} \frac{\pi}{\xi} \leq 1, \\
\left|b_{k}^{*}(\xi)\right| & \leq\left.\frac{2 \xi}{\pi} \cdot \frac{1}{\xi} \operatorname{arctg} \frac{u}{\xi}\right|_{0} ^{\infty}=1, \\
\left|c_{k}(\xi)\right| & \leq \frac{4 \xi^{3}}{\pi} \int_{0}^{\infty} \frac{d u}{\left(u^{2}+\xi^{2}\right)^{2}}=1,
\end{aligned}
$$

which immediately implies (i). 
(ii) First, it is immediate that we can write

$$
Q_{\xi}(f)(z)=\frac{\xi}{\pi} \int_{-\pi}^{\pi} \frac{f\left(z e^{-i u}\right)}{u^{2}+\xi^{2}} d u .
$$

Since $h(u)=1 /\left(u^{2}+\xi^{2}\right)$ satisfies $h(\pi)=h(-\pi)$, we may extend it by $2 \pi$-periodicity on the whole $\mathbb{R}$, such that this extension is continuous on $\mathbb{R}$.

By $h^{\prime}(u)=-2 u /\left(u^{2}+\xi^{2}\right)^{2}$, it follows that $h$ is nondecreasing on $[-\pi, 0]$ and nonincreasing on $[0, \pi]$. Then, by $\left[11\right.$, Theorem 3, page 799], it follows that $Q_{\xi}(f)(z)$ is closeto-convex on $D$.

(iii) Let $f \in \mathscr{P}, f=U+i V, U>0$. Then, by definitions, it easily follows that $Q_{\xi}(f)$, $Q_{\xi}^{*}(f), R_{\xi}(f) \in \mathscr{P}$. We take here into account that, by Theorem 3.1(i), the condition $a_{0}=f(0)=1$ implies

$$
\begin{gathered}
Q_{\xi}^{*}(f)(0)=a_{0} b_{0}^{*}(\xi)=b_{0}^{*}(\xi)=\frac{\xi}{\pi} \int_{-\infty}^{+\infty} \frac{d u}{u^{2}+\xi^{2}}=1 \\
R_{\xi}(f)(0)=a_{0} c_{0}(\xi)=\frac{2 \xi^{3}}{\pi} \int_{-\infty}^{+\infty} \frac{d u}{\left(u^{2}+\xi^{2}\right)^{2}}=1
\end{gathered}
$$

Now, let $f(z)=\sum_{k=0}^{\infty} a_{k} z^{k}$, with $a_{0}=0, a_{1}=1$. First, by Theorem 3.1(i), we get

$$
\begin{array}{ccc}
\frac{1}{b_{1}(\xi)} Q_{\xi}(f)(0)=0, & \frac{1}{b_{1}(\xi)} Q_{\xi}^{\prime}(f)(0)=1, & \frac{1}{b_{1}^{*}(\xi)} Q_{\xi}^{*}(f)(0)=0, \\
\frac{1}{b_{1}^{*}(\xi)} \cdot\left[Q_{\xi}^{*}(f)\right]^{\prime}(0)=1, & \frac{1}{c_{1}(\xi)} R_{\xi}(f)(0)=0, & \frac{1}{c_{1}(\xi)} \cdot R_{\xi}^{\prime}(f)(0)=1 .
\end{array}
$$

Then,

$$
\begin{gathered}
Q_{\xi}^{\prime \prime}(f)(z)=\frac{\xi}{\pi} \int_{-\pi}^{\pi} f^{\prime \prime}\left(z e^{i u}\right) e^{2 i u} \cdot \frac{1}{u^{2}+\xi^{2}} d u, \\
{\left[Q_{\xi}^{*}(f)\right]^{\prime \prime}(z)=\frac{\xi}{\pi} \int_{-\infty}^{+\infty} f^{\prime \prime}\left(z e^{-i u}\right) e^{-2 i u} \cdot \frac{1}{u^{2}+\xi^{2}} d u} \\
{\left[R_{\xi}(f)\right]^{\prime \prime}(z)=\frac{2 \xi^{3}}{\pi} \int_{-\infty}^{+\infty} f^{\prime \prime}\left(z e^{i u}\right) e^{2 i u} \cdot \frac{1}{\left(u^{2}+\xi^{2}\right)^{2}} d u .}
\end{gathered}
$$

Let $f \in S_{3, b_{1}(\xi)}$. We get

$$
\begin{aligned}
\left|\frac{1}{b_{1}(\xi)} \cdot Q_{\xi}^{\prime \prime}(f)(z)\right| & \leq \frac{1}{\left|b_{1}(\xi)\right|} \cdot \frac{\xi}{\pi} \int_{-\pi}^{\pi}\left|f^{\prime \prime}\left(z e^{i u}\right)\right| \cdot \frac{1}{u^{2}+\xi^{2}} d u \\
& \leq \frac{\xi}{\pi} \int_{-\pi}^{\pi} \frac{d u}{u^{2}+\xi^{2}}=\frac{2}{\pi} \operatorname{arctg} \frac{\pi}{\xi} \leq 1,
\end{aligned}
$$

that is, $\left(1 / b_{1}(\xi)\right) \cdot Q_{\xi}(f) \in S_{3}$. 


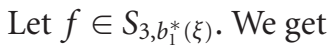

$$
\begin{aligned}
\left|\frac{1}{b_{1}^{*}(\xi)} \cdot\left[Q^{*}(f)\right]^{\prime \prime}(z)\right| & \leq \frac{1}{\left|b_{1}^{*}(\xi)\right|} \cdot \frac{\xi}{\pi} \int_{-\infty}^{+\infty}\left|f^{\prime \prime}\left(z e^{i u}\right)\right| \cdot \frac{1}{u^{2}+\xi^{2}} d u \\
& \leq \frac{\xi}{\pi} \int_{-\infty}^{+\infty} \frac{d u}{u^{2}+\xi^{2}}=1
\end{aligned}
$$

that is, $\left(1 / b_{1}^{*}(\xi)\right) Q_{\xi}^{*}(f) \in S_{3}$. The proof in the case of $\left(1 / c_{1}(\xi)\right) \cdot R_{\xi}(f)$ is similar.

Now, let $f \in S_{M}$. It follows that

$$
\begin{aligned}
\left|\frac{1}{b_{1}(\xi)} Q_{\xi}^{\prime}(f)(z)\right| & \leq \frac{1}{\left|b_{1}(\xi)\right|} \frac{\xi}{\pi} \int_{-\pi}^{\pi}\left|f^{\prime}\left(z e^{i u}\right)\right| \cdot \frac{1}{u^{2}+\xi^{2}} d u \\
& <\frac{M}{\left|b_{1}(\xi)\right|} \cdot \frac{2}{\pi} \operatorname{arctg} \frac{\pi}{\xi} \leq \frac{M}{\left|b_{1}(\xi)\right|} .
\end{aligned}
$$

The proofs in the cases of $\left(1 / b_{1}^{*}(\xi)\right) \cdot Q_{\xi}^{*}(f)$ and $\left(1 / c_{1}(\xi)\right) \cdot R_{\xi}(f)$ are similar, which proves the theorem.

Remarks 3.3. (1) Theorem 3.2(iii) says that if $f \in S_{3, b_{1}(\xi)}$, then $Q_{\xi}(f)$ is starlike and univalent on $D$ and if $f \in S_{M /\left|b_{1}(\xi)\right|}$, then $Q_{\xi}(f)$ is univalent in the disk

$$
\left\{z \in \mathbb{C} ;|z|<\frac{\left|b_{1}(\xi)\right|}{M}\right\} \subset\left\{z \in \mathbb{C} ;|z|<\frac{1}{M}\right\} .
$$

Similar properties hold for $Q_{\xi}^{*}(f), b_{1}^{*}(\xi)$, and $R_{\xi}(f), c_{1}(\xi)$.

(2) Let us denote $B=\inf \left\{\left|b_{1}(\xi)\right| ; \xi \in(0,1]\right\}$. If $B>0$, then, by Theorem 3.2(iii), the following properties hold: $f \in S_{3, B}$ implies $Q_{\xi}(f) \in S_{3}$, for all $\xi \in(0,1], f \in S_{M}(M>1)$ implies that $Q_{\xi}(f)$ is univalent in $\{|z|<B / M\}$, for all $\xi \in(0,1]$. Therefore it remains to calculate $B$, to check if $B>0$, problems which are left to the reader as open questions.

Now, since $\inf \left\{\left|b_{1}^{*}(\xi)\right| ; \xi \in(0,1]\right\}=\inf \left\{e^{-\xi} ; \xi \in(0,1]\right\}=1 / e$ and $\inf \left\{\left|c_{1}(\xi)\right| ; \xi \in\right.$ $(0,1]\}=\inf \left\{(1+\xi) e^{-\xi} ; \xi \in(0,1]\right\}=2 / e\left(\right.$ since $h(\xi)=(1+\xi) e^{-\xi}$ is decreasing on $\left.[0,1]\right)$, from Theorems 3.1(i) and 3.2(iii), we immediately get the following.

Corollary 3.4. (i) If $f \in S_{3,1 / \text { e }}$, then $Q_{\xi}^{*}(f) \in S_{3}$, for all $\xi \in(0,1]$, and if $f \in S_{M}(M>1)$, then $Q_{\xi}^{*}(f)$ is univalent in $\{z \in \mathbb{C} ;|z|<1 / e M\}$, for all $\xi \in(0,1]$.

(ii) If $f \in S_{3,2 / e}$, then $R_{\xi}(f) \in S_{3}$, for all $\xi \in(0,1]$, and if $f \in S_{M}$, then $R_{\xi}(f)$ is univalent in $\{|z|<2 / e M\}$, for all $\xi \in(0,1]$.

\section{Complex Gauss-Weierstrass integrals}

In this section, we study the complex integrals $W_{\xi}(f)(z)$ and $W_{\xi}^{*}(f)(z)$.

Concerning the approximation properties, we present the following.

TheOREM 4.1. (i) If $f(z)=\sum_{k=0}^{\infty} a_{k} z^{k}$ is analytic in $D$, then for all $\xi>0, W_{\xi}(f)(z)$ and $W_{\xi}^{*}(f)(z)$ are analytic in $D$ and the following holds on $D$ :

$$
W_{\xi}(f)(z)=\sum_{k=0}^{\infty} a_{k} d_{k}(\xi) z^{k},
$$


with

$$
\begin{gathered}
d_{k}(\xi)=\frac{1}{\sqrt{\pi \xi}} \cdot \int_{-\pi}^{\pi} e^{-u^{2} / \xi} \cos k u d u, \\
W_{\xi}^{*}(f)(z)=\sum_{k=0}^{\infty} a_{k} d_{k}^{*}(\xi) z^{k},
\end{gathered}
$$

with

$$
d_{k}^{*}(\xi)=\frac{1}{\sqrt{\pi \xi}} \int_{-\infty}^{+\infty} e^{-u^{2} / \xi} \cos k u d u
$$

Also, if $f$ is continuous on $\bar{D}$, then $W_{\xi}(f)$ and $W_{\xi}^{*}(f)$ are continuous on $\bar{D}$. Here $d_{1}(\xi)>0$ and $d_{1}^{*}(\xi)=e^{-\xi / 4} \cdot 1 / \pi$, for all $\xi>0$.

(ii)

$$
\begin{aligned}
& \left|W_{\xi}(f)(z)-f(z)\right| \leq C \frac{\omega_{2}(f ; \xi) \partial D}{\xi}, \quad z \in \bar{D}, \xi \in(0,1], \\
& \left|W_{\xi}^{*}(f)(z)-f(z)\right| \leq C \omega_{1}(f ; \sqrt{\xi})_{\bar{D}}, \quad z \in \bar{D}, \xi \in(0,1] .
\end{aligned}
$$

(iii)

$$
\begin{array}{ll}
\omega_{1}\left(W_{\xi}^{*}(f) ; \delta\right)_{\bar{D}} \leq \omega_{1}(f ; \delta)_{\bar{D}}, & \forall \delta>0, \xi>0, \\
\omega_{1}\left(W_{\xi}(f) ; \delta\right)_{\bar{D}} \leq \omega_{1}(f ; \delta)_{\bar{D}}, & \forall \delta>0, \xi>0 .
\end{array}
$$

Proof. (i) Reasoning as for the $P_{\xi}(f)$ operator, we can write

$$
\begin{aligned}
W_{\xi}^{*}(f)(z) & =\frac{1}{\sqrt{\pi x}} \int_{-\infty}^{+\infty} \sum_{k=0}^{\infty} a_{k} z^{k} e^{i u k} e^{-u^{2} / \xi} d u \\
& =\sum_{k=0}^{\infty} a_{k} z^{k} \cdot \frac{1}{\sqrt{\pi \xi}} \int_{-\infty}^{+\infty}[\cos (k u)+i \sin (k u)] e^{-u^{2} / \xi} d u=\sum_{k=0}^{\infty} a_{k} d_{k}^{*}(\xi) z^{k}
\end{aligned}
$$

where

$$
d_{k}^{*}(\xi)=\frac{1}{\sqrt{\pi \xi}} \int_{-\infty}^{+\infty} \cos (k u) e^{-u^{2} / \xi} d u
$$

The reasonings in the case of $W_{\xi}(f)(z)$ are similar. The proof of continuity on $\bar{D}$ of $W_{\xi}(f)$ and $W_{\xi}^{*}(f)$ is similar to that for $P_{\xi}(f)$ in the proof of Theorem 2.1(i).

It remains to prove that $d_{1}(\xi)>0$, for all $\xi>0$, and that $d_{1}^{*}(\xi)=(1 / \pi) e^{-\xi / 4}$, for all $\xi>0$.

Indeed, firstly we have

$$
d_{1}(\xi)=\frac{1}{\sqrt{\pi \xi}} \int_{-\pi}^{\pi} e^{-u^{2} / \xi} \cos u d u=\frac{2}{\sqrt{\pi} \eta} \int_{0}^{\pi} \cos u e^{-(u / \eta)^{2}} d u,
$$


where $\eta=\sqrt{\xi}>0$. We obtain

$$
\begin{aligned}
d_{1}(\eta) & =\frac{2}{\sqrt{\pi} \eta} \cdot\left[\int_{0}^{\pi / 2} \cos u e^{-(u / \eta)^{2}} d u+\int_{\pi / 2}^{\pi} \cos u e^{-(u / \eta)^{2}} d u\right] \\
& =\frac{2}{\sqrt{\pi} \eta}\left[\int_{0}^{\pi / 2} \cos u e^{-(u / \eta)^{2}} d u-\int_{0}^{\pi / 2} \sin u e^{-((u+\pi / 2) / \eta)^{2}} d u\right] \\
& >\frac{2}{\sqrt{\pi} \eta}\left[\int_{0}^{\pi / 2}(\cos u-\sin u) e^{-(u / \eta)^{2}} d u\right]:=\frac{2}{\sqrt{\pi} \eta}\left[I_{1}+I_{2}\right],
\end{aligned}
$$

where

$$
\begin{gathered}
I_{1}=\int_{0}^{\pi / 4}(\cos u-\sin u) e^{-(u / \eta)^{2}} d u>0, \\
I_{2}=-\int_{\pi / 4}^{\pi / 2}(\sin u-\cos u) e^{-(u / \eta)^{2}} d u<0 .
\end{gathered}
$$

It follows that

$$
\begin{gathered}
I_{1}>\int_{0}^{\pi / 4}(\cos u-\sin u) e^{-(\pi /(4 \eta))^{2}} d u=(\sqrt{2}-1) e^{-(\pi /(4 \eta))^{2}} \\
\left|I_{2}\right|=-I_{2}<e^{-(\pi /(4 \eta))^{2}} \int_{\pi / 4}^{\pi / 2}(\sin u-\cos u) d u=(\sqrt{2}-1) e^{-(\pi / 4 \eta))^{2}}
\end{gathered}
$$

Therefore,

$$
d_{1}(\eta)>I_{1}+I_{2} \geq(\sqrt{2}-1) e^{-(\pi /(4 \eta))^{2}}-(\sqrt{2}-1) e^{-(\pi /(4 \eta))^{2}}=0,
$$

for any $\eta>0$.

Now, for $d_{1}^{*}(\xi)=(1 / \sqrt{\pi} \sqrt{\xi}) \cdot \int_{-\infty}^{+\infty} \cos u e^{-(u / \sqrt{\xi})^{2}} d u$, we have (see, e.g., [10, page 228])

$$
\begin{gathered}
\frac{1}{\sqrt{\pi} \sqrt{\xi}} \cdot \int_{-\infty}^{+\infty} \cos u e^{-(u / \sqrt{\xi})^{2}} d u=\left(\text { by } \frac{u}{\sqrt{\xi}}=v\right)=\frac{1}{\sqrt{\pi}} \int_{-\infty}^{+\infty} \cos (\sqrt{\xi} v) e^{-v^{2}} d v \\
=\frac{1}{\sqrt{\pi}} \int_{-\infty}^{+\infty} e^{i \sqrt{\xi} v} e^{-v^{2}} d v=\frac{1}{\sqrt{\pi}} \cdot e^{-\xi / 4} \cdot \frac{1}{\sqrt{\pi}}=\frac{e^{-\xi / 4}}{\pi},
\end{gathered}
$$

for all $\xi>0$.

(ii) We can write

$$
\begin{aligned}
W_{\xi}(f)(z)-f(z)= & \frac{1}{\sqrt{\pi \xi}} \int_{0}^{\pi}\left[f\left(z e^{i u}\right)-2 f(z)+f\left(z e^{-i u}\right)\right] e^{-u^{2} / \xi} d u \\
& +f(z)\left[1-\frac{1}{\sqrt{\pi \xi}} \int_{-\pi}^{\pi} e^{-u^{2} / \xi} d u\right] .
\end{aligned}
$$


Here

$$
\begin{aligned}
\left|f(z)\left[1-\frac{1}{\sqrt{\pi \xi}} \cdot \int_{-\pi}^{\pi} e^{-u^{2} / \xi} d u\right]\right| \\
\quad=\left|f(z)\left[1-\frac{2}{\sqrt{\pi \xi}} \int_{0}^{\pi} e^{-u^{2} / \xi} d u\right]\right| \\
=\left|f(z)\left[\frac{2}{\sqrt{\pi \xi}} \int_{0}^{\infty} e^{-u^{2} / \xi} d u-\frac{2}{\sqrt{\pi \xi}} \int_{0}^{\pi} e^{-u^{2} / \xi} d u\right]\right| \\
=|f(z)| \cdot\left|\frac{2}{\sqrt{\pi \xi}} \int_{\pi}^{\infty} e^{-u^{2} / \xi} d u\right| \\
\leq\|f\|_{\bar{D}} \cdot \frac{2}{\sqrt{\pi \xi}} \int_{\pi}^{\infty} \frac{\xi}{u^{2}} d u=2\|f\|_{\bar{D}} \sqrt{\xi} \cdot \frac{1}{\pi \sqrt{\pi}} .
\end{aligned}
$$

By the maximum modulus principle, we can take $|z|=1$ which implies

$$
\begin{aligned}
\left|W_{\xi}(f)(z)-f(z)\right| & \leq \frac{1}{\sqrt{\pi \xi}} \int_{0}^{\pi} \omega_{2}(f ; u)_{\partial D} e^{-u^{2} / \xi} d u+2\|f\|_{\bar{D}} \sqrt{\xi} \frac{1}{\pi \sqrt{\pi}} \\
& (\text { reasoning as in }[2 \text {, page 258]) } \\
& \leq \frac{C \omega_{2}(f ; \xi)_{\partial D}}{\xi}+2\|f\|_{\bar{D}} \sqrt{\xi} \cdot \frac{1}{\pi \sqrt{\pi}} \leq C \frac{\omega_{2}(f ; \xi)_{\partial D}}{\xi} .
\end{aligned}
$$

Also, we get

$$
\begin{aligned}
\left|W_{\xi}^{*}(f)(z)-f(z)\right| & \leq \frac{1}{\sqrt{\pi \xi}} \int_{-\infty}^{+\infty}\left|f\left(z e^{-i u}\right)-f(z)\right| e^{-u^{2} / \xi} d u \\
& \leq \frac{1}{\sqrt{\pi \xi}} \int_{-\infty}^{\infty} \omega_{1}\left(f ;\left|1-e^{-i u}\right|\right)_{\bar{D}} e^{-u^{2} / \xi} d u \\
& =\frac{1}{\sqrt{\pi \xi}} \int_{-\infty}^{+\infty} \omega_{1}\left(f ; 2\left|\sin \frac{u}{2}\right|\right)_{\bar{D}} e^{-u^{2} / \xi} d u \\
& \leq \frac{1}{\sqrt{\pi \xi}} \int_{-\infty}^{+\infty} \omega_{1}(f ;|u|)_{\bar{D}} e^{-u^{2} / \xi} d u \\
& \leq \frac{1}{\sqrt{\pi \xi}} \int_{-\infty}^{+\infty} \omega_{1}(f ; \sqrt{\xi})_{\bar{D}}\left(\frac{|u|}{\sqrt{\xi}}+1\right) e^{-u^{2} / \xi} d u \\
& =\omega_{1}(f ; \sqrt{\xi})_{\bar{D}}+\frac{\omega_{1}(f ; \sqrt{\xi})_{\bar{D}}}{\sqrt{\xi} \cdot \sqrt{\pi \xi}} 2 u \int_{0}^{-u^{2} / \xi} d u .
\end{aligned}
$$


But $\int_{0}^{\infty} 2 u e^{-u^{2} / \xi} d u=\xi \int_{0}^{\infty} e^{-v} d v=\xi$, which implies

$$
\left|W_{\xi}^{*}(f)(z)-f(z)\right| \leq \omega_{1}(f ; \sqrt{\xi})_{\bar{D}}+\omega_{1}(f ; \sqrt{\xi})_{\bar{D}} \cdot \frac{\xi}{\xi \sqrt{\pi}} \leq C \omega_{1}(f ; \sqrt{\xi})_{\bar{D}} .
$$

(iii) For $\left|z_{1}-z_{2}\right|<\delta$, we get

$$
\begin{aligned}
\left|W_{\xi}^{*}(f)\left(z_{1}\right)-W_{\xi}^{*}(f)\left(z_{2}\right)\right| & \leq \frac{1}{\sqrt{\pi \xi}} \cdot \int_{-\infty}^{+\infty}\left|f\left(z_{1} e^{-i u}\right)-f\left(z_{2} e^{-i u}\right)\right| e^{-u^{2} / \xi} d u \\
& \leq \omega_{1}\left(f ;\left|z_{1}-z_{2}\right|\right)_{\bar{D}} \leq \omega_{1}(f ; \delta)_{\bar{D}} \\
\left|W_{\xi}(f)\left(z_{1}\right)-W_{\xi}(f)\left(z_{2}\right)\right| & \leq \frac{1}{\sqrt{\pi \xi}} \int_{-\pi}^{+\pi}\left|f\left(z_{1} e^{i u}\right)-f\left(z_{2} e^{i u}\right)\right| e^{-u^{2} / \xi} d u \\
& \leq \omega_{1}\left(f ;\left|z_{1}-z_{2}\right|\right)_{\bar{D}} \cdot \frac{1}{\sqrt{\pi \xi}} \int_{-\pi}^{\pi} e^{-u^{2} / \xi} d u \\
& \leq \omega_{1}(f ; \delta)_{\bar{D}} \cdot \frac{1}{\sqrt{\pi \xi}} \int_{-\infty}^{+\infty} e^{-u^{2} / \xi} d u=\omega_{1}(f ; \delta)_{\bar{D}},
\end{aligned}
$$

which proves the theorem.

Concerning the geometric properties of complex Gauss-Weierstrass singular integrals, we present the following.

Theorem 4.2. (i) If $f(z)=\sum_{k=0}^{\infty} a_{k} z^{k}, z \in D$, and $T_{\xi}(f)(z)=\sum_{k=0}^{\infty} A_{k} z^{k}$ is any from $W_{\xi}(f)(z)$ and $W_{\xi}^{*}(f)(z)$, then

$$
\left|A_{k}\right| \leq\left|a_{k}\right|, \quad \forall k=0,1, \ldots
$$

(ii) If $f(z)=\sum_{k=1}^{\infty} a_{k} z^{k}, z \in D$, is univalent in $D$ and $f(D)$ is convex, then for any $\xi>0$, $W_{\xi}(f)(z)$ is univalent in $D$ and $W_{\xi}(f)(D)$ is convex.

Similarly, if $f(z)$ is univalent in $D$ and $f(D)$ is starlike with respect to the origin, then for any $\xi>0, W_{\xi}(f)(z)$ is univalent in $D$ and $W_{\xi}(f)(D)$ is starlike with respect to the origin.

(iii) For all $\xi>0$, with the notations in Theorem 3.2,

$$
\begin{gathered}
W_{\xi}^{*}(\mathscr{P}) \subset \mathscr{P}, \quad \frac{1}{d_{1}(\xi)} W_{\xi}\left(S_{3, d_{1}(\xi)}\right) \subset S_{3}, \\
\frac{1}{d_{1}^{*}(\xi)} W_{\xi}^{*}\left(S_{3, d_{1}^{*}(\xi)}\right) \subset S_{3}, \quad \frac{1}{d_{1}(\xi)} W_{\xi}\left(S_{M}\right) \subset S_{M /\left|d_{1}(\xi)\right|} \\
\frac{1}{d_{1}^{*}(\xi)} W_{\xi}^{*}\left(S_{M}\right) \subset S_{M /\left|d_{1}^{*}(\xi)\right|}
\end{gathered}
$$


Proof. (i) By Theorem 4.1(i), we get

$$
\begin{aligned}
\left|a_{k} d_{k}(\xi)\right| & \leq\left|a_{k}\right| \cdot\left|d_{k}(\xi)\right| \leq\left|a_{k}\right| \cdot \frac{1}{\sqrt{\pi \xi}} \int_{-\pi}^{\pi} e^{-u^{2} / \xi}|\cos k u| d u \\
& \leq\left|a_{k}\right| \cdot \frac{1}{\sqrt{\pi \xi}} \int_{-\infty}^{+\infty} e^{-u^{2} / \xi} d u=\left|a_{k}\right|, \quad \forall k=0,1,2, \ldots
\end{aligned}
$$

Also, by the same theorem, we obtain

$$
\left|a_{k} d_{k}^{*}(\xi)\right|=\left|a_{k}\right| \cdot\left|d_{k}^{*}(\xi)\right| \leq\left|a_{k}\right|, \quad \forall k=0,1,2, \ldots
$$

Also, note that $\left|d_{0}(\xi)\right|=d_{0}(\xi) \leq 1$ and $\left|d_{0}^{*}(\xi)\right|=d_{0}(\xi)=1$.

(ii) Let $g(u)=e^{-u^{2} / \xi}, u \in[-\pi, \pi]$. Since $g(-\pi)=g(\pi)$, we can extend $g(u)$ by $2 \pi$ periodicity on the whole $\mathbb{R}$, such that the extension, denoted by $h(u)$, is continuous on $\mathbb{R}$.

It is easy to check that $\log \left|h^{\prime}(u)\right|$ is concave in each interval $[k \pi,(k+1) \pi], h^{\prime}(u)=0$ if and only if $u=2 k \pi, k \in \mathbb{Z}$, and in $u_{k}=k \pi, k \in \mathbb{Z}, h$ takes its minimum and maximum values.

Then, applying [9, Theorem, page 130], we get that $h$ is PMP as in [9], which implies that $W_{\xi}(f)$ preserves the convexity of $f$.

Also, by similar reasoning with those in [8, Lemma 5 and Corollary 5, page 321], it follows that $W_{\xi}(f)(z)$ preserves the starlikeness of $f(z)$ (with respect to origin) too.

(iii) The proofs are similar to the proofs in Theorem 3.2(iii), which proves Theorem 4.2 too.

Remarks 4.3. (1) From the results presented above, it follows that $W_{\xi}(f)(z)$ has the best preservation property among the classes of complex singular integrals studied by the present paper.

(2) Let us denote $D=\inf \left\{\left|d_{1}(\xi)\right| ; \xi \in(0,1]\right\}$. If $D>0$, then, by Theorem 4.2(iii), we get the following:

(i) if $f \in S_{3, D}$ then $W_{\xi}(f) \in S_{3}$, for all $\xi \in(0,1]$,

(ii) if $f \in S_{M},(M>1)$, then $W_{\xi}(f)$ is univalent in $\{z \in \mathbb{C}$; $|z|<D / M\}$, for all $\xi \in$ $(0,1]$.

Therefore it remains to calculate $D$, to check if $D>0$, problems which are left to the reader as an open question.

Since $\inf \left\{\left|d_{1}^{*}(\xi)\right| ; \xi \in(0,1]\right\}=1 /\left(\pi e^{1 / 4}\right)$, applying now Theorems 4.1(i) and 4.2(iii) to $W_{\xi}^{*}(f)(z)$, we immediately get the following.

Corollary 4.4. If $f \in S_{3,1 / \pi e^{1 / 4}}$, then $W_{\xi}^{*}(f) \in S_{3}$, for all $\xi \in(0,1]$, and if $f \in S_{M}(M>1)$, then $W_{\xi}^{*}(f)$ is univalent in $\left\{z \in \mathbb{C} ;|z|<1 / \pi M e^{1 / 4}\right\}$, for all $\xi \in(0,1]$.

\section{Acknowledgment}

This paper was written during the 2005 Spring Semester when the second author was a Visiting Professor at the Department of Mathematical Sciences, University of Memphis, Tenn, USA. 


\section{References}

[1] J. W. Alexander, Functions which map the interior of the unit circle upon simple regions, Annals of Mathematics. Second Series 17 (1915), no. 1, 12-22.

[2] S. G. Gal, Degree of approximation of continuous functions by some singular integrals, Revue d'Analyse Numérique et de Théorie de l'Approximation 27 (1998), no. 2, 251-261.

[3] Convolution-type integral operators in complex approximation, Computational Methods and Function Theory 1 (2001), no. 2, 417-432.

[4] - On the Beatson convolution operators in the unit disk, Journal of Analysis 10 (2002), 101-106.

[5] _ Geometric and approximate properties of convolution polynomials in the unit disk, Bulletin of the Institute of Mathematics. Academia Sinica 1 (2006), no. 2, 307-336.

[6] P. T. Mocanu, T. Bulboaca, and Gr. St. Salagean, Geometric Theory of Univalent Functions, Casa Cartii de Stiinta, Cluj, 1999.

[7] M. Obradović, Simple sufficient conditions for univalence, Matematichki Vesnik 49 (1997), no. 3$4,241-244$.

[8] G. Pólya and I. J. Schoenberg, Remarks on de la Vallée Poussin means and convex conformal maps of the circle, Pacific Journal of Mathematics 8 (1958), no. 2, 295-334.

[9] S. Ruscheweyh and L. C. Salinas, On the preservation of periodic monotonicity, Constructive Approximation 8 (1992), no. 2, 129-140.

[10] I. Stewart and D. Tall, Complex Analysis, Cambridge University Press, Cambridge, 2002.

[11] T. J. Suffridge, Convolutions of convex functions, Journal of Mathematics and Mechanics 15 (1966), 795-804.

George A. Anastassiou: Department of Mathematical Sciences, University of Memphis, Memphis, TN 38152, USA

E-mail address: ganastss@memphis.edu

Sorin G. Gal: Department of Mathematics, University of Oradea, Str. Armatei Romane 5,

Oradea 410087, Romania

E-mail address: galso@uoradea.ro 